\title{
Correlation of Chest CT and RT-PCR Testing for Coronavirus Disease 2020(COVID-19) in Palestine: A Report of 200 Cases
}

\author{
Sami Smerat, Murad Abu Samra, Maram Ghassan Sada, Azzam Alarab, Issa Al Hroush, \\ Moath Al-Makhamreh, Osama Najjar, and Mohammed Hjouj
}

\section{ABSTRACT}

Purpose: Prove that Computed Tomography (CT) is a more effective diagnostic tool for diagnosing Coronavirus Disease 2019 (COVID-19) in comparison with Reverse Transcription Polymerase Chain Reaction (RTPCR) due to its higher sensitivity.

Methods and materials: A total of 200 cases that had records of chest CT scans and RT-PCR tests were collected from Palestinian governmental hospitals from September to November 2020.

Results: CT indicated COVID-19 in $62.5 \%$ of sample population (125 of 200) whereas RT-PCR indicated COVID-19 in 37.5\% (75 of 200) cases. A $P$-value of 0.028 signifies the existence of a relationship between gender and CT sensitivity.

Conclusion: High resolution computed tomography (HRCT) of the chest is a more rapid, sensitive, and effective tool than the current gold standard (RT-PCR); therefore, should be considered a primary diagnostic method for COVID-19.

Keywords: COVID-19, CT, RT-PCR, Sensitivity.

Submitted : December 28, 2020

Published : April 23, 2021

ISSN: $2593-8339$

DOI: $10.24018 /$ ejmed.2021.3.2.634

Mohammed Hjouj*

Al-Quds University, Palestine.

(e-mail: mhjouj@staff.alquds.edu)

Sami Smerat

Istishari Arab Hospital, Palestine.

(e-mail: ssmerat ${ }^{\circledR}$ paluniv.edu.ps)

Murad Abu Samra

Icahn School of Medicine at Mount Sinai, NY, USA.

(e-mail: muradabusamra ${ }^{@}$ gmail.com)

Maram Ghassan Sada

Al-Quds University, Palestine.

(e-mail: maram.sada ${ }^{@}$ students.alquds.edu) Azzam Alarab

Palestine Ahliya University, Palestine.

Issa Sameeh Al Hroush

Istishari Arab Hospital, Palestine.

Moath Al-Makhamreh

Yatta Public Hospital, Palestine.

Osama Najjar

Palestinian Ministry of Health, Palestine.

*Corresponding Author

\section{INTRODUCTION}

The first cases infected with severe acute respiratory syndrome coronavirus 2 virus (SARS-CoV-19) appeared in Wuhan, Hubei Province, China in December of 2019. Weeks later, the virus spread rapidly across China and the rest of the world [1]. The rising numbers of confirmed cases necessitated international measures to help contain the spread. These measures included but were not limited to diagnostic testing, management of confirmed cases, and community Infection Prevention and Control (IPC) [1]. Considering that the identification of infected people is the first and most crucial step to containing the spread, a diagnostic tool that is available, rapid, sensitive, and affordable is essential [2]. The current diagnostic test for Coronavirus Disease 2019 (COVID-19) is Reverse transcription polymerase chain reaction (RT-PCR) assay [3]. Through oropharyngeal (OP) or nasopharyngeal (NP) swabs, specimens are obtained to detect the presence of SARS-CoV2 [4]. As previously mentioned, early detection of COVID-
19 is essential to prevent and control infectious transmission; however, many issues are presented with the use of RT-PCR as the gold standard of testing [5]. The waiting time, limited availability, and costly nature renders the RT-PCR as an ineffective diagnostic tool [4]. The most concerning issue of using RT-PCR is its inability to detect the viral genome of symptomatic or asymptomatic people (5). Specifically, the sensitivity of RT-PCR is very limited (60\%-71\%) [5]. The first factor that limits the sensitivity of RT-PCR assays as a diagnostic tool is the inherent susceptibility to errors due to specimen collection [4]. There are many methods to collect specimens, but the most rapid and common are NP and/ or OP swabs, and improper sample collection, transportation, and/or handling can result in inconsistencies [5]. Moreover, RT-PCR is vulnerable to inter-operator variability that can affect the quality of the collected sample [5]. Finally, the limited sensitivity of RT-PCR is also due to its dependence upon viral load, sampling timing, and period of the disease development [5]. A diagnostic tool that is considered to be effective due to its availability, high sensitivity, and rapidity 
is chest computed tomography (CT) scan. Chest CT scans reveal anatomic details and convey the extent of pulmonary damage due to COVID-19. Known patterns of imaging manifestations of COVID-19 have been indicated on chest CT scans, such as: Ground Glass Opacities (GGOs), pulmonary consolidations, reticular patterns, and pleural thickening [6]. Despite its important role in the COVID-19 pandemic, chest CT is being used as an adjunct to RT-PCR [7]. Due to the urgency of the situation and the need of an effective diagnostic tool, this study aims at assessing the sensitivity of RT-PCR and chest CT scans in the diagnosis of COVID-19.

\section{Methods AND Materials}

\section{A. Study Population}

This retrospective cohort study included 200 patients with confirmed COVID-19. 100 patients (50\%) were female and $100(50 \%)$ were male. Samples were collected from Palestinian governmental hospitals from September to November 2020. Information regarding the type of diagnostic test performed and the gender were extracted through electronic medical records. The entire sample population was included in the study according to the following inclusion criteria:

1) each patient performed both chest CT scan and RT-PCR assay.

2) one of the diagnostic tests indicated a positive COVID19 result.

\section{B. Ethical Consideration}

Approval was acquired from the Research Ethics Committee of the Palestinian Ministry of Health (MoH).

\section{Statistical Analysis}

The SPSS 24.0 software was used to study the difference in RT-PCR and CT. Descriptive and frequency statistics was used to study the main characteristic of the sample. Continuous variables were given as mean \pm standard deviation while categorical variables were given as number and percentage. The Kruskal-Wallis test and Mann-Whitney $\mathrm{U}$ test were included in the analysis.

\section{RESULTS}

00 patients participated in this study. 100 patients $(50 \%)$ were male, and 100 patients $(50 \%)$ were female. The entire sample population underwent both diagnostic tests (Fig. 1). CT confirmed COVID-19 in 62.5\% (125/200) of the population while RT-PCR confirmed COVID-19 in $37.5 \%$ (75/200) of population. Of the 125 patients that received a positive result through CT, 55 were female while 70 were male. Additionally, of the 75 patients that received a positive RT-PCR, 45 were female and 30 were male (Fig. 2).

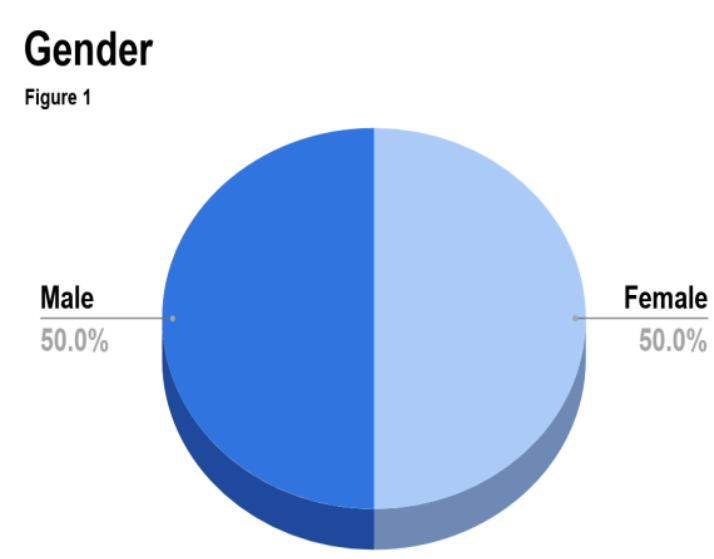

Fig. 1. Pie chart of gender.

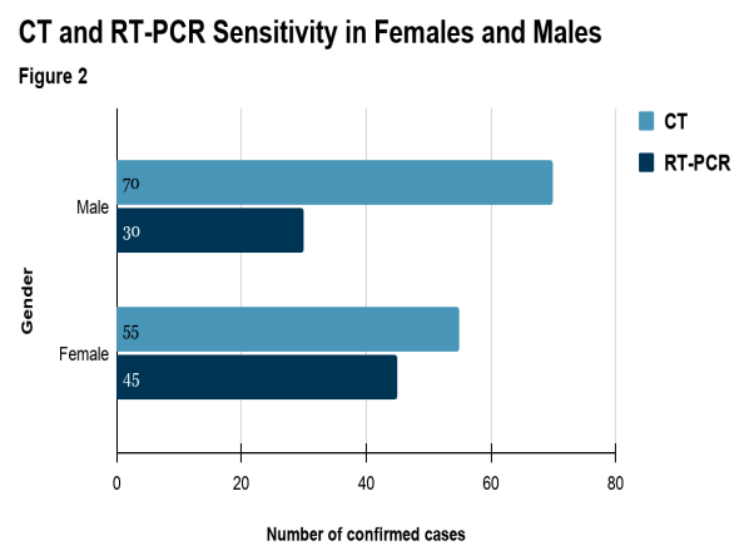

Fig. 2. Bar graph of confirmed cases using RT-PCR and CT. According to the two-tailed $P$-value: 0.028 , there is a significant relationship between gender and CT sensitivity, i.e., the sensitivity varies for males and females (the results in Fig. 2. reflects this relationship).

\section{DISCUSSION}

Coronavirus disease 2019(COVID-19) is a highly infectious disease that is caused by SARS-CoV-2 [1]. The first cases of this worldwide pandemic originated in Hubei Province, China and were reported in December 2019 [1]. Due to the rapid spread and increase in mortality rates, practical, available, and sensitive methods of diagnosis are needed. Early diagnosis of COVID-19 plays a pivotal role in controlling the transmission of this highly infectious viral agent. The current gold standard for diagnosing COVID-19 is reverse-transcription polymerase chain reaction RT-PCR; however, recent studies expose the lack of sensitivity and unreliability of using RT-PCR as the reference diagnosticCOVID-19 test [3]. Despite its use as the gold standard to diagnose COVID-19, limitations such as long processing time, susceptibility to inherent procedural errors, inter-operator variability, and inherent erroneous results due to sample collection and analysis decrease the reliability of RT-PCR [5]. On the other hand, chest CT scans of the thorax show promise in diagnosing COVID-19. CT is rapid, costeffective, and available rendering it a more sensitive and practical method of diagnosing COVID-19. Through viewing the extent of pulmonary damage through crosssectional anatomical details, one can determine whether the patient has contracted COVID-19 [6]. 
According to studies that evaluate the imaging patterns of COVID-19 such as the study conducted by Chung et al., CT findings such as Ground Glass Opacities (GGOs) were present in $73 \%$ of patients [8]. Additionally, a pictorial review conducted by Ye et al. reported that CT has a sensitivity of 98\% when used to conclude the presence of COVID-19 [9]. Moreover, Long et al. revealed in his study that the sensitivity of CT was $92.7 \%$ whereas the sensitivity of RT-PCR was $83.3 \%$ [10]. A study conducted by Ai et al. revealed that the sensitivity of CT was $97.2 \%$, whereas the sensitivity of RTPCR was $83.3 \%$. According to these results, our study agreed with previous studies [11].

Our retrospective analysis showed similar results. Our analysis consisted of 200 patients who were tested for COVID-19 through both diagnostic tools (RT-PCR and chest $\mathrm{CT}$ ); the sensitivity of chest CT scans was far greater than that of RT-PCR. Specifically, the sensitivity of RT-PCR test reported to be $37.5 \%$ (75 of 200 patients). On the contrary, CT indicated COVID-19 in $62.5 \%$ of the patients (125 of 200). The decreased sensitivity of RT-PCR is thought to be due to the test's susceptibility to yield false negative and false-positive results due to its dependence upon viral load, improper sample collection, and sample analysis. Falsenegative results can be detrimental in a pandemic due to the importance of early detection and action. A $P$ - value of .028 emphasizes the existence of a significant relationship between gender and CT sensitivity.

The Mann-Whitney U-test and Kruskal-Wallis $\mathrm{H}$ test indicated that there was a significant relationship between gender and the result of CT scans as well. Meaning, the sensitivity of CT was slightly less in females than that for males.

\section{CONCLUSION}

Finally, our study strongly supports the use of high resolution chest CT scans for patients with suspected COVID-19 pneumonia. Because the use of RT-PCR can prevent early diagnosis due to its decreased sensitivity, a diagnostic test that can instigate early action is necessary. Rapid diagnosis performed through chest CT can lead to early action and increase public health care services. Overall, the speed, sensitivity, and availability of CT renders it a more effective diagnostic tool for testing the presence of COVID19 in comparison to RT-PCR

\section{REFERENCES}

[1] Li, Y. \&amp; Xia, L. Coronavirus disease 2019 (COVID-19): Role of chest CT in diagnosis and management. Am. J. Roentgenol. (2020) doi:10.2214/AJR.20.22954.

[2] Bai, H. X. et al. Performance of radiologists in differentiating COVID19 from viral pneumonia on chest CT. Radiology (2020) doi:10.1148/radiol.2020200823.

[3] Fang, Y. et al. Sensitivity of Chest CT for COVID-19: Comparison to RT-PCR. Radiology (2020) doi:10.1148/radiol.2020200432.

[4] Tang, Y. W., Schmitz, J. E., Persing, D. H. \&amp; Stratton, C. W. Laboratory diagnosis of COVID-19: Current issues and challenges. Journal of Clinical Microbiology.

[5] Tahamtan, A. \&amp; Ardebili, A. Real-time RT-PCR in COVID-19 detection: issues affecting the results. Expert Review of Molecular Diagnostics (2020) doi:10.1080/14737159.2020.1757437.
[6] Li, X. et al. CT imaging changes of coronavirus disease 2019(COVID-19): A multi-center study in Southwest China. J. Transl. Med. (2020) doi:10.1186/s12967-020-02324-w.

[7] Ye, Z., Zhang, Y., Wang, Y., Huang, Z. \&amp; Song, B. Chest CT manifestations of new coronavirus disease 2019 (COVID-19): a pictorial review. Eur. Radiol. (2020) doi:10.1007/s00330-020-068010 .

[8] M. Chung et al., "CT imaging features of 2019 novel coronavirus (2019-NCoV)," Radiology, vol. 295, no. 1. pp. 202-207, 2020, doi: 10.1148/radiol.2020200230.

[9] Z. Ye, Y. Zhang, Y. Wang, Z. Huang, and B. Song, "Chest CT manifestations of new coronavirus disease 2019 (COVID-19): a pictorial review," European Radiology, vol. 30, no. 8. pp. 4381-4389, 2020, doi: 10.1007/s00330-020-06801-0.

[10] Long, C., Xu, H., Shen, Q., Zhang, X., Fan, B., Wang, C., Li, H. (2020). Diagnosis of Coronavirus disease (COVID-19): rRT-PCR or CT? European Journal of Radiology. https://doi.org/10.1016/j.ejrad.2020.108961.

[11] Ai, T. et al. Correlation of Chest CT and RT-PCR Testing for Coronavirus Disease 2019 (COVID-19) in China: A Report of 1014 Cases. Radiology (2020) doi:10.1148/radiol.2020200642.

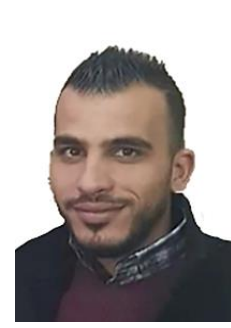

Sami Smerat was born in the city of Hebron, Palestine. He received his B.A. degree in Radiography from Palestine Ahliya university in Bethlehem, Palestine in 2016. Moreover, he was a Research assistant (RA) and Lecturer in Palestine Ahliya university for four years. He has worked as a Radiologic technologist in various private clinics and is currently working at Istishari Arab Hospital. He is a proactive researcher and has published research regarding diagnostic imaging and physiotherapy. His areas of interest are physiotherapy and ultrasound.

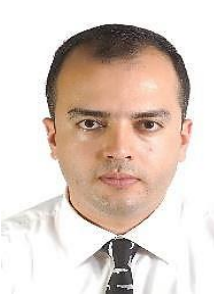

Murad Mohammad Abu Samra was born in the city of Hebron in Palestine. He received his doctor of medicine degree (M.D.) from Al-Quds University in 2007. Moreover, he earned his specialty in Diagnostic radiology from ACGME-I program in the American University of Beirut in Lebanon in 2016. Afterwards, he specialized in Nuclear oncology from the Memorial Sloan Kettering Cancer Center in New York, USA in 2019 and in 2020 he specialized in MRI Oncologic Imaging. Currently, he is working as an Assistant professor in Icahn School of Medicine at Mount Sinai, New York, USA. Previously, he worked as a teaching assistant in the anatomy department at Al-Quds university in 2011. He earned extensive working and training experience as a radiologist and specialized nuclear oncologist in multiple clinics and hospitals. He has published many articles involving breast cancer imaging through PET/CT, interventional imaging, and radiation protection involving nuclear medicine.

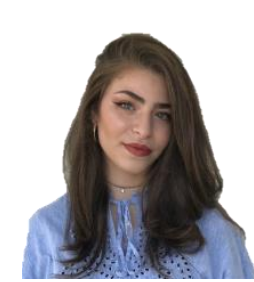

Maram Ghassan Sada was born in Chicago, Illinois in the United States of America. She is currently earning her B.A. degree in Radiography from Al-Quds University. She participated in an internship job as a Radiologic technologist (R.T.) at Al-Lijan Imaging Center in Hebron. She previously conducted unpublished research involving the risk perception of medical imaging. She is currently conducting research regarding the common imaging manifestations of COVID-19 in which she is planning on publishing. Her research ambitions include hybrid imaging, cardiac imaging, and artificial intelligence (AI).

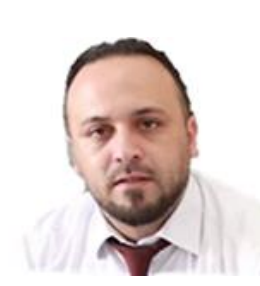

Azzam Alarab was born in the city of Hebron in Palestine. He earned a B.A. degree in Physical therapy from the Arab American University in 2008. Afterwards, he received his M.A. degree in Physiotherapy and rehabilitation from Dokuz Eylul university in Turkey in the year 2013. Moreover, he received his $\mathrm{PhD}$ in Orthopedic rehabilitation from Pamukkale university in Turkey. He has published many articles, one of which evaluates the therapeutic techniques for musculoskeletal pain. 


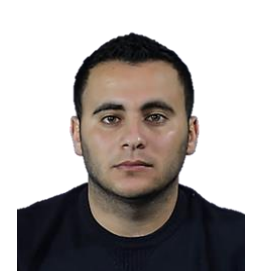

imaging sections

Issa Sameeh Al Hroush was born in Hebron, Palestine. He earned a B.A. degree in Radiography from the Arab American university in the year 2013. He previously worked as a Radiologic technologist (R.T.) at the Palestinian Red Crescent Society. He was Chief technologist and training coordinator at An-Najah University Hospital. He is currently chief radiologic technologist at the Istishari Arab Hospital where he is specialized in and oversees CT and MRI

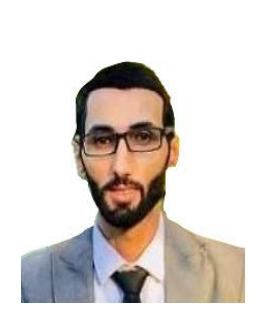

Moath Al-Makhamreh was born in Hebron, Palestine. He earned his B.A. degree in Radiography from Al-Quds university in 2011. Afterwards, he received a M.A. degree from Al-Quds university in Functional imaging in the year 2020. He previously worked as a Radiologic technologist (R.T.) at Al-Ahli Hospital in Hebron. Currently, he works at Public Yatta Hospital as a Radiologic Technologist (R.T.). $\mathrm{He}$ published an article regarding IV contrast timing in abdominal CT imaging.

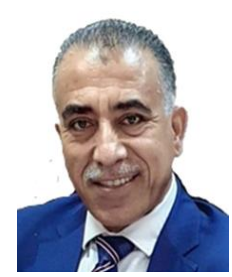

Osama Najjar was born in Hebron, Palestine. He received his B.A. degree in Clinical laboratory science from Al-Quds university in 1993. Afterwards, he earned his M.A. degree in Clinical laboratory from Birzeit university in 2007. Moreover, he is currently receiving his Ph.D. in Hematology. Furthermore, he currently is the head of health professions in the Palestinian Ministry of Health and Laboraty Medical Sector. His research interests include but are not limited to hematology, analytical chemistry, and quality control in labs.

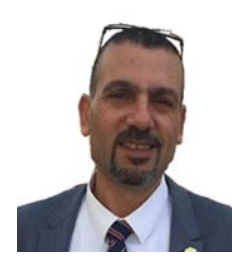

Mohammed Hjouj was born in Hebron, Palestine. He received a B.A. degree in Radiography from Al-Quds university in the year 2002. Later in 2004 he earned his M.A. degree in Medical imaging from the University of Leeds in the UK. Additionally, he earned his Ph.D. in Biomedical engineering and medical imaging from The Hebrew university in Jerusalem in 2013. He is currently a professor in the medical imaging department of Al-Quds university; moreover, he was previously the head of the department. He is an academic and PACS administrator at Al-Makassed Hospital in Jerusalem. He has participated in a plethora of training programs involving MRI safety, radiation safety, and ultrasonography (to mention a few). Furthermore, he is an active member of international and local conferences and workshops that focus on scientific research. Throughout his lengthy professional experience as M.A. coordinator and researcher, he has published articles involving MR Imaging, hepatocellular carcinoma, image-guided therapy, etc. His research interests aim at developing affordable medical diagnosis and treatment technologies to reduce medical care cost in remote and impoverished parts of the world including: Cancer Diagnosis and Treatment, electroporation (Reversible: ECT \&amp; EGT, and Irreversible). medical imaging technology Functional Imaging mainly (fMRI, EEG, MEG, NM), and osteoporosis. $\mathrm{He}$ is a member of the International Society for Electroporation-Based Technologies and Treatments (ISEBTT) and The European Society of Radiology (ESR). 